\title{
La verdadera nave Torroja de Villaverde
}

\section{The true Eduardo Torroja's hall in Villaverde}

\author{
R. García ${ }^{(*)}$
}

\section{RESUMEN}

Este trabajo resume la investigación realizada mediante la cual se ha podido probar que Eduardo Torroja es autor de una de las naves industriales de la antigua empresa Boetticher y Navarro de Villaverde en Madrid. La nave en cuestión, destinada originalmente a talleres, es contigua a la conocida como nave Catedral, hoy rehabilitada como Centro de Nuevas Tecnologías del Ayuntamiento de Madrid. El interés de esta edificación no está solo en la autoría sino también en su solución estructural, innovadora en España en los años en que se proyectó y construyó (1940-42). La solución se describe y analiza en relación a datos de otras construidas posteriormente y de carácter semejante. Afortunadamente además, la nave se encuentra en pie y en uso, aunque con otro cometido que el original y perteneciente a otra empresa. Su documentación gráfica se publica aquí por primera vez.

Palabras clave: Torroja, Boetticher, prefabricado hormigón, cercha de hormigón, arquitectura industrial, Villaverde.

\section{ABSTRACT}

This paper summarizes the research by which has been able to prove that engineer Eduardo Torroja authored one of the industrial halls of the old complex Boetticher \& Navarro in Villaverde, Madrid. Originally it was used as mechanical workshops. The hall is close to the today famous "nave Catedral", restored in recent years as New Technologies Centre of the Madrid Council. The interest of this hall no only lies in the authorship but also in its structural solution, innovative in Spain in the years in which was designed and built (1940-1942). The structural system is described and compared with other similar structures built later. Fortunately, the structure is still standing, but with a different purpose than the original and belonging to another company. The original plans of the "true" Boetticher \& Navarro Torroja hall are published here for the first time.

Keywords: Torroja, Boetticher, prefab concrete, concrete truss, industrial architecture, Villaverde

(*) Universidad Politécnica de Madrid (España)

Persona de contacto/Corresponding author: rafael.garcia@upm.es (R. García)

ORCID: http://orcid.org/oooo-0oo2-9237-8291 (R. García)

Cómo citar este artículo/Citation: R. García (2018). La verdadera nave Torroja de Villaverde. Informes de la Construcción, 70(551): e267. https://doi.org/10.3989/ic.59350

Copyright: ( $\mathbf{2 0 1 8}$ CSIC. Este es un artículo de acceso abierto distribuido bajo los términos de la licencia de uso y distribución Creative Commons Reconocimiento 4.o Internacional (CC BY 4.0). 


\section{INTRODUCCIÓN}

Durante mucho tiempo los vecinos del distrito madrileño de Villaverde, tuvieron como atribuida al ingeniero Eduardo Torroja la nave llamada Catedral o nave Boetticher, destacada por su singularidad dentro del conjunto de las antiguas instalaciones de la empresa de construcciones metálicas Boetticher y Navarro. Con su reconocible silueta de sección basilical abovedada ha tenido siempre un claro protagonismo visual acaparando la atención de todas las miradas incluso después de perder su uso industrial. Hoy sabemos que dicha atribución era errónea y que su autor fue el ingeniero de la empresa Manuel Cámara Muñoz (Figura 1).

Con todo, la confusión ha sido frecuente para esta nave que incluso llegó a ser popularmente conocida como "nave Torroja” y que aun en informaciones periodísticas recientes aparece referida como obra de Torroja. Por ejemplo, en $L a$ Vanguardia 22/04/2016 13:48 ed. online se dice: "Diseñada en la década de los años 40 por el ingeniero Eduardo Torroja" o también en El Mundo 25/01/2016 23:25 ed. online, Catedral de las Nuevas Tecnologías "La construcción industrial de Villaverde, que ahora se reinaugurará como Catedral de las Nuevas Tecnologías, fue diseñada en 1934 (sic) por el ingeniero Eduardo Torroja.

Sin embargo, la investigación que aquí exponemos en detalle, nos ha permitido constatar que aunque efectivamente esa nave no fue diseñada por Torroja, éste sí realizó un proyecto construido de nave para dicha empresa, pero en ubicación diferente, lo cual al parecer ha pasado inadvertido hasta el momento, sin que ninguna publicación haya dado referencia precisa de ello. Lo más sorprendente es que dicha nave está aún en pie, es de considerables dimensiones y se encuentra justo delante de la unánimemente apreciada nave Catedral. No se trata por tanto de una nave menor o marginal sino que se trata de la primera gran nave construida para dicha empresa en Villaverde y destinada originalmente a talleres mecánicos. Su uso actual, segregada totalmente de la anterior entidad, es de garaje de la empresa de autobuses SAMAR SA.

Respecto a la nave Catedral, no fue hasta la realización de un estudio reciente con motivo de su conversión en Centro de Nuevas Tecnologías promovido por el Ayuntamiento de Madrid, que se pudo encontrar que su autoría correspondía al mencionado ingeniero Manuel Cámara Muñoz, quien la proyectó en 1949. Dicho informe con el título Investigación y diagnóstico de los procesos patológicos del edificio Boetticher, Villaverde, Madrid, consta de dos partes, una documental y otra un estudio técnico, y está en posesión del Área de Gobierno de Economía y Participación Ciudadana del Ayuntamiento de Madrid, la cual encargo su realización al Instituto de Ciencias de la Construcción Eduardo Torroja (información facilitada a través del servicio de biblioteca del Instituto). Una parte de sus resultados fueron expuestos en forma de paneles con fotografías en la exposición sobre el "Convenio urbanístico para la transformación de las instalaciones de Boetticher en Villaverde" organizada en la misma Nave Catedral en abril de 2009 (1).

Por otra parte y en nuestras propias investigaciones independientes hemos podido constatar además, que este mismo ingeniero realizó para el mismo conjunto fabril varios otros proyectos notables de naves también con abovedados de hormigón para almacenes, garaje y taller de carpintería y modelos, todas ellas demolidas (2). Al menos las dos primeras mencionadas, de almacenes y garaje, se construyeron incluso antes que la nave Catedral, después adosada a ellas.

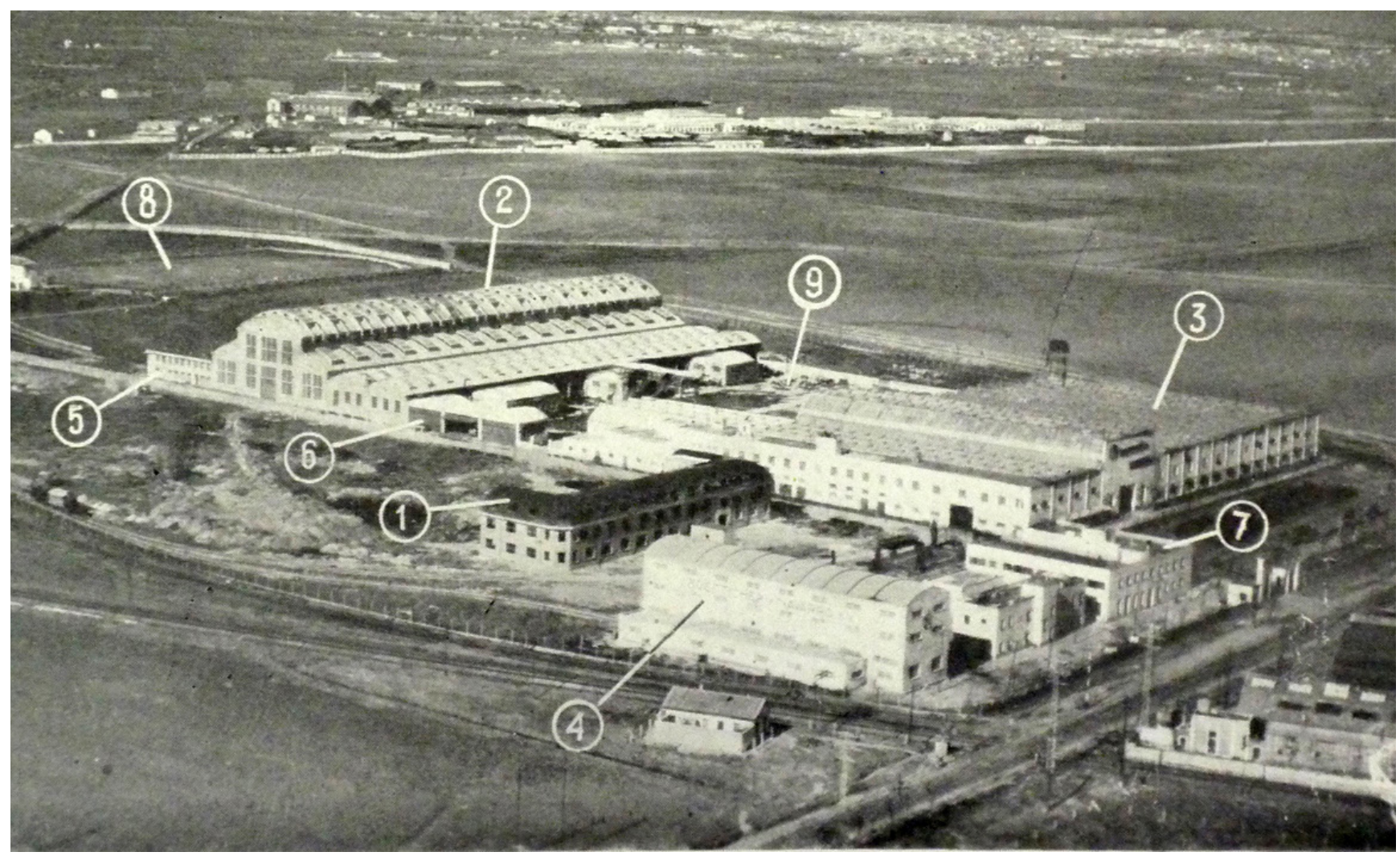

Figura 1. Complejo Boetticher y Navarro. Comienzos años 50. Número 2, nave Catedral, número 3, nave de talleres (INI 1952 p. 7). 


\section{INVESTIGACIÓN DOCUMENTAL}

Pero volviendo a la nave de talleres mecánicos objeto de nuestro estudio, se ha de resaltar que aunque exteriormente es menos atractiva por su aparente sencillez formal, existe en ella un rasgo distintivo que fue el que atrajo nuestra atención y motivó la búsqueda documental. ${ }^{1}$ Este es la llamativa presencia exterior en cubierta de grandes cordones exentos uniendo grupos de dientes de sierra, lo que hacía suponer, antes de un examen del interior no fácil por su carácter privado, la existencia de luces interiores mayores de las habituales y por tanto cierto carácter inconvencional. La nave parecía ofrecer por tanto, algún interés especial y animar a realizar algunas indagaciones. Y efectivamente, pronto surgieron las primeras pruebas de su singularidad.

Un dibujo en perspectiva publicado por la asociación vecinal de Villaverde, El Espinillo, nos presenta precisamente esta nave ya terminada junto a algunas otras construcciones previstas para la primera fase de la fábrica, pero sin que aparezca todavía la nave Catedral ni sus naves abovedadas anexas hoy desaparecidas (Figura 2). La figura se publicó con el siguiente comentario: "Vista general de las instalaciones de Boetticher y Navarro (junto con la Fundición Iglesias) y antes de la construcción de la nave "Torroja" o "la Catedral" en 1947", con ello se abunda por tanto en el error de atribución. En cuanto a la mencionada fundición Iglesias, ésta se asoció con Gómez Navarro al finalizar la guerra pero fue absorbida pronto por ella. Los talleres que se representan en el dibujo a su nombre y con la misma solución que la nave de talleres, nunca se construyeron (3). Según este dibujo, se habría construido antes que ninguna otra, lo cual parece tener cierta lógica por su posición más adelantada hacia la carretera de Andalucía. Esto se ha visto efectivamente corroborado en el expediente sobre esta nave, finalmente encontrado en el Archivo de la Villa de Madrid con fecha de 1940 (4). Dicho expediente es por otra parte, la prueba definitiva de la autoría, ya que dentro de él, el proyecto aparece firmado en julio de ese mismo año por el arquitecto Ricardo Gómez Abad y el ingeniero Eduardo Torroja (Figura 3). Otro elemento probatorio de su anticipación temporal es una fotografía en la revista Gran Madrid en donde se aprecian en construcción en primer plano las naves menores con bóvedas de hormigón antes mencionadas, y detrás y sobresaliendo por encima, el perfil de dientes de sierra de la nave, ya construida (5).

El rasgo fundamental de esta "nueva" o "verdadera" si se quiere, nave Torroja, es como se ha indicado, la estructura de cubierta, lo cual se aprecia explícitamente en el proyecto depositado en el Ayuntamiento (Figura 4). Fue realizada con cerchas de hormigón armado de tipo Warren asimétrico lo que también se puede entender como formadas por la unión,

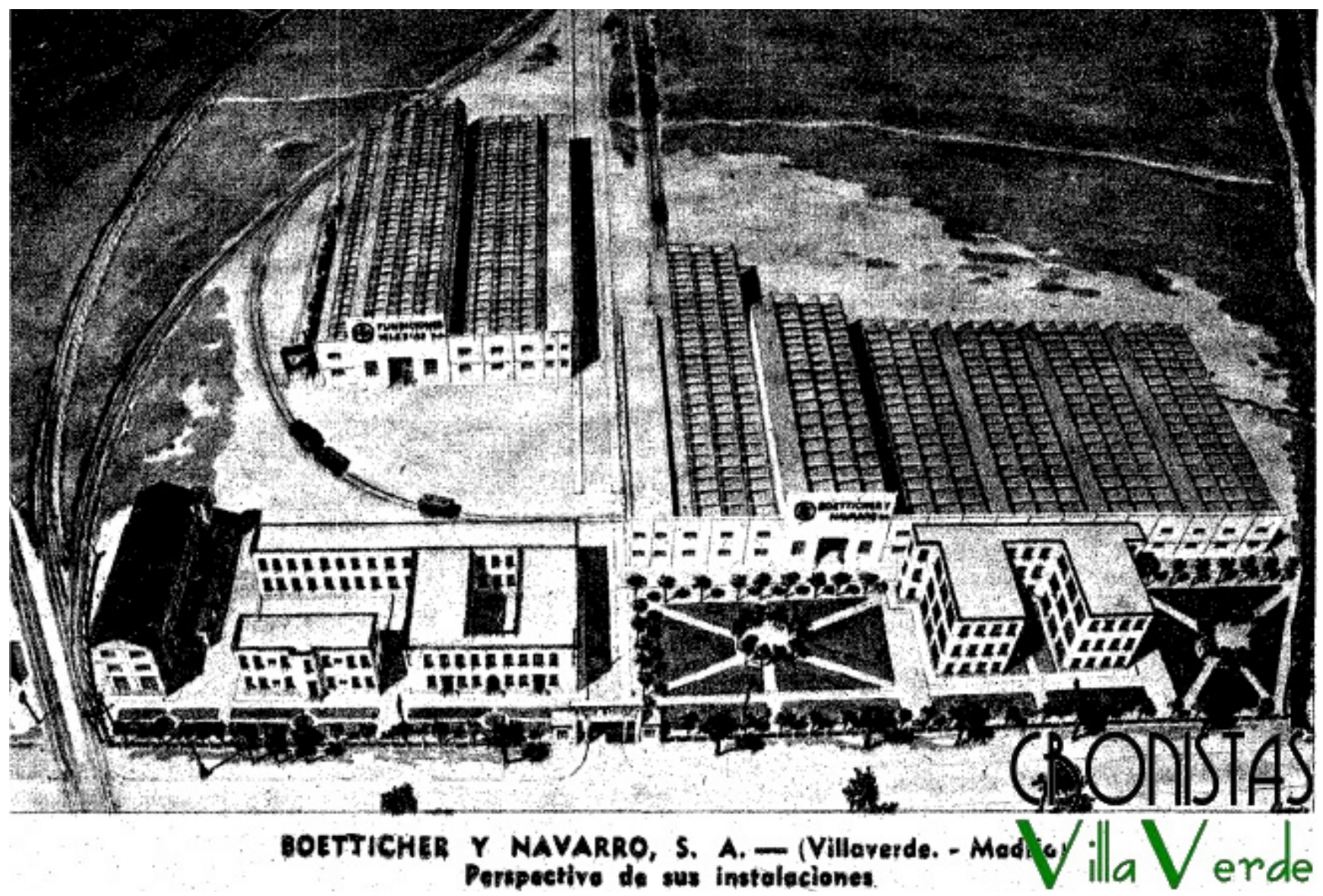

Figura 2. Nave de talleres de E. Torroja. La nave de la izquierda no fue construida. 1947 (asociación El Espinillo).

\footnotetext{
La investigación se enmarca dentro del contexto de un estudio más amplio de quien esto suscribe, todavía en curso de realización, sobre edificaciones industriales aún no catalogadas en el ámbito madrileño. Un estado previo de la investigación sobre la nave de talleres se presentó en las XVIII Jornadas INCUNA de Patrimonio Industrial en septiembre de 2016 en forma parcial y junto con otros edificios industriales madrileños de interés y poco conocidos.
} 


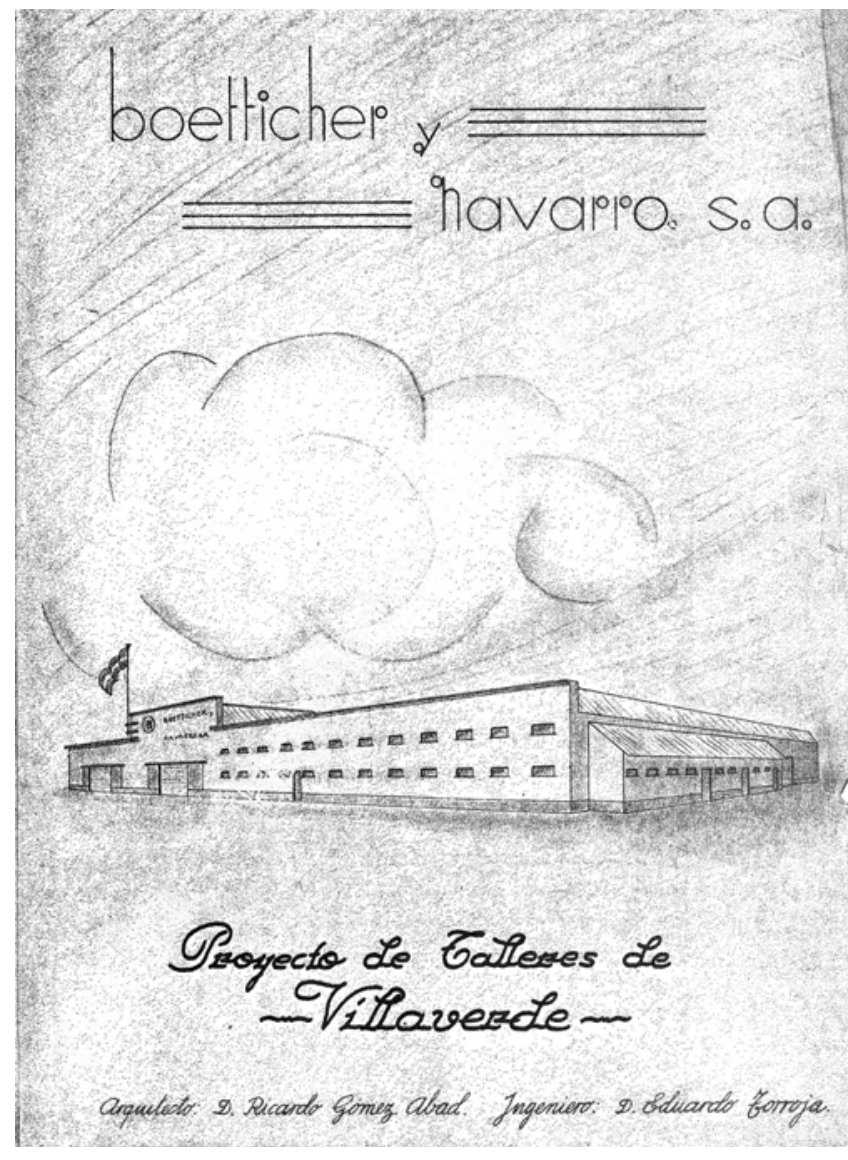

Figura 3. Proyecto de Talleres de Villaverde. Portada con nombres de autores (AVM exp. 33-332-1).

mediante un cordón superior, de los triángulos de cuatro unidades de diente de sierra. Estas cerchas, con luces de $20 \mathrm{~m}$, fueron prefabricadas en obra y en realidad se adelantan a todas las por nosotros conocidas de este tipo con cordones paralelos en España, las cuales se realizaron algo después, cuando se fue generalizando esta práctica. Destacan además por su esbeltez y por superar a las subsiguientes de estas mismas características en luz y número de tramos. Es notable además que la obra se llevara a cabo a tan poco tiempo del final de la guerra civil, ya que su inauguración como nuevos talleres de la sociedad en Villaverde tuvo lugar el 30 de noviembre de 1942 (6). La planta cubierta inicialmente por dicha nave fue de seis vanos de $20 \mathrm{~m}$ todos ellos con longitud de $76 \mathrm{~m}$, o sea un área de 120 x 76 m (Figura 5).

Sobre este punto existe también una nueva fuente de confirmación, ya que en el archivo Torroja del CEHOPU, se encuen- tra un conjunto de proyectos de 1940 realizados por encargo de talleres Gómez Navarro y coincidentes con la nave objeto de este artículo. Es llamativo que aunque el proyecto real y los otros estudios previos existentes en CEHOPU están adecuadamente registrados (ETM-212-001 y ETM-212-002, exp. 394), no se hayan asociado con la nave correcta. Así por ejemplo, en el extenso trabajo de referencia sobre las estructuras de edificación de Torroja de J. Antuña (7), se citan los talleres Gómez Navarro, exp. $\mathrm{n}^{0} 394$ del A.E.T [Archivo Eduardo Torroja], capítulo 5, pero se los asocia con la nave Catedral. En otros trabajos de importancia dedicados a Torroja y en los que se incluye una visión panorámica de su obra, como los de J.A. Fernández Ordóñez y Navarro Vera (8), C. Jordá (9), Levi (10), P. Chías (11) o el del mismo Torroja en explicación de su propia obra (12) no se encuentra ninguna referencia a esta nave. Únicamente en el libro de Arredondo y Verdú (13) publicado en 1977 existe una referencia nominal en su listado de obras en la que en página 335 se indica "Talleres Boetticher y Navarro en Madrid”, pero sin más datos ni consignación de fecha. Esa misma referencia vuelve a repetirse literalmente sin más en el catálogo de la exposición celebrada en el Colegio de Ingenieros de Caminos, Canales y Puertos de Madrid en 1979, página 111 (14).

En los mencionados estudios previos se aprecia en primer lugar que la planta considerada viene a coincidir con bastante exactitud con la realizada, aunque su rectángulo tenía una crujía más - 7 frente a las 6 construidas (Figura 6) (15). También difiere la crujía de mayor altura y situada en segundo lugar desde la izquierda en el sentido del plano, que en el estudio previo tenía más luz que el resto, pero finalmente cubrió igual ancho.

Tiene interés comentar dichas propuestas previas, en tanto que nos dan idea de cómo Torroja fue barajando y descartando alternativas que en sí mismas eran bastante originales. Las llegó a detallar bastante minuciosamente, la mayoría con dibujos acotados, y varias ofrecen soluciones de cubiertas shed o diente de sierra, planteadas sobre la misma planta general (16). Estas fueron: un original diseño de bóvedas laminares de un tercio de la luz y apoyadas en tornapuntas; bóvedas cilíndricas convencionales de todo el ancho (Figura 7); y variantes de pórticos en diente de sierra, rectos y arqueados, todo ello en hormigón. Estas cuatro soluciones previas indicadas e incluso otra más con cuchillos metálicos también contemplada, están denominadas en el archivo con las letras A, B y C correspondientes a tres tipos de cubierta: A, teja sobre forjado de hormigón, B, Uralita sobre cielo raso de corcho y $\mathrm{C}$, teja sobre bóveda de hormigón. Estas letras corresponden a los tipos indicados en los planos, pero llama

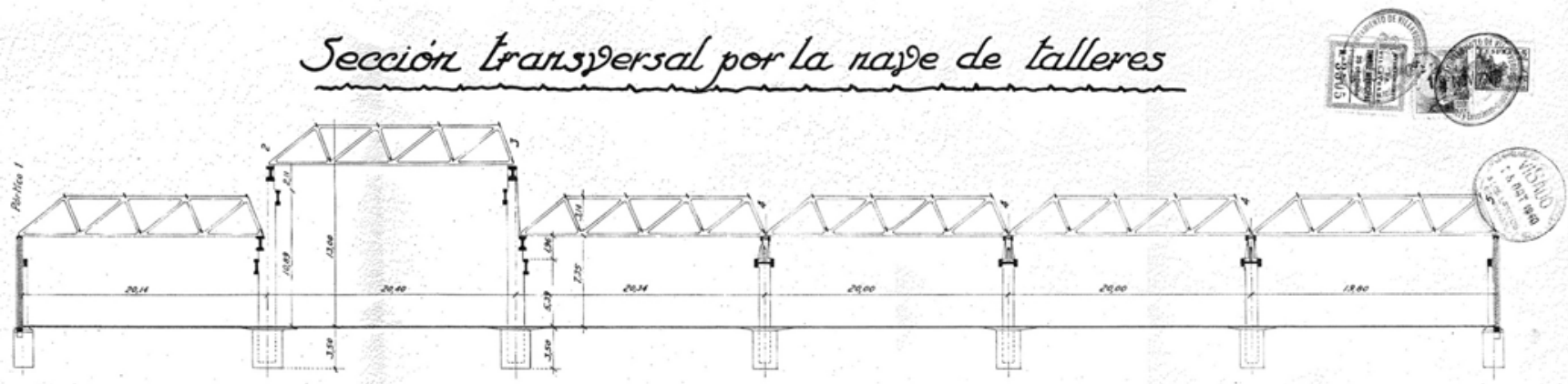

Figura 4. Sección transversal nave talleres (AVM exp. 33-332-1). 


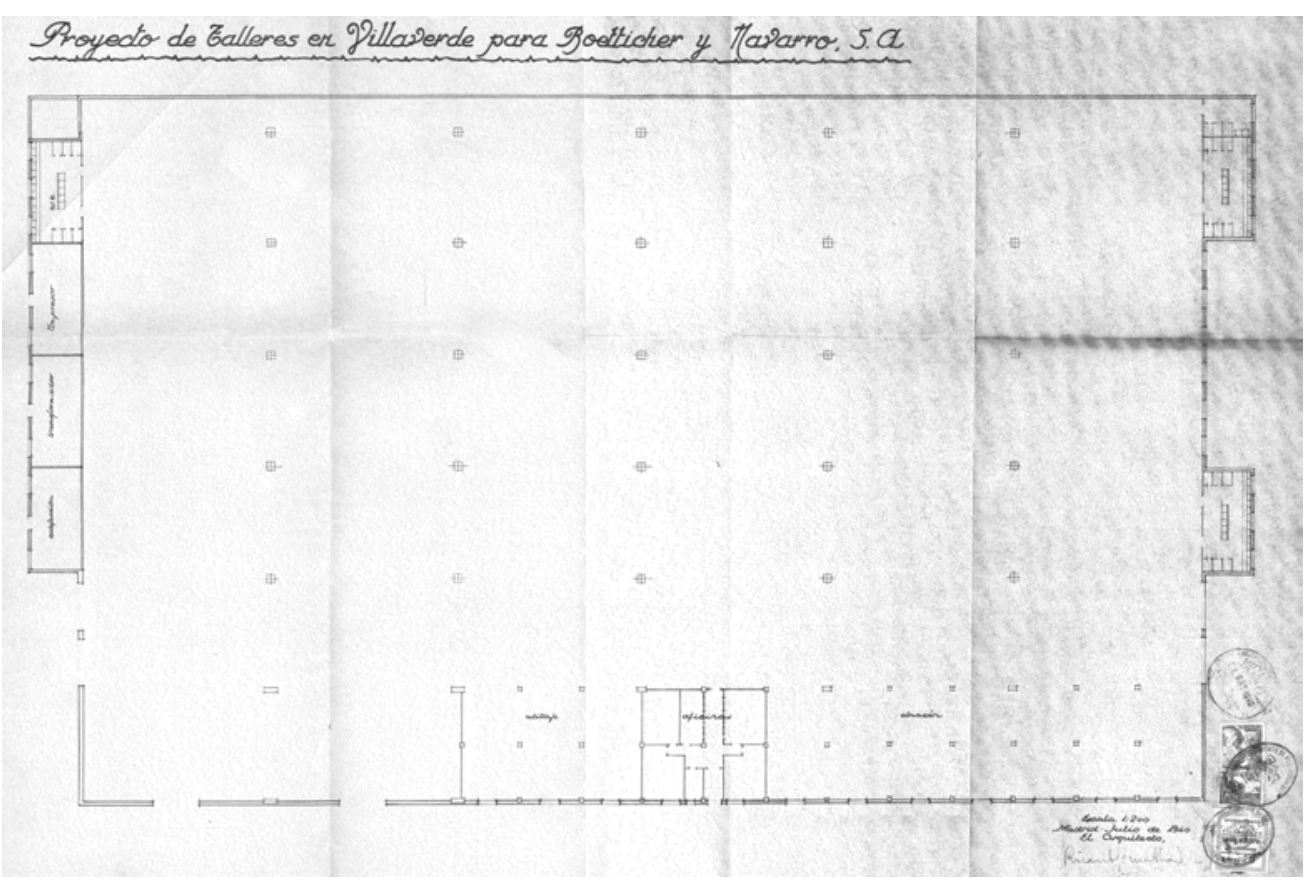

Figura 5. Planta nave talleres (AVM exp. 33-332-1).

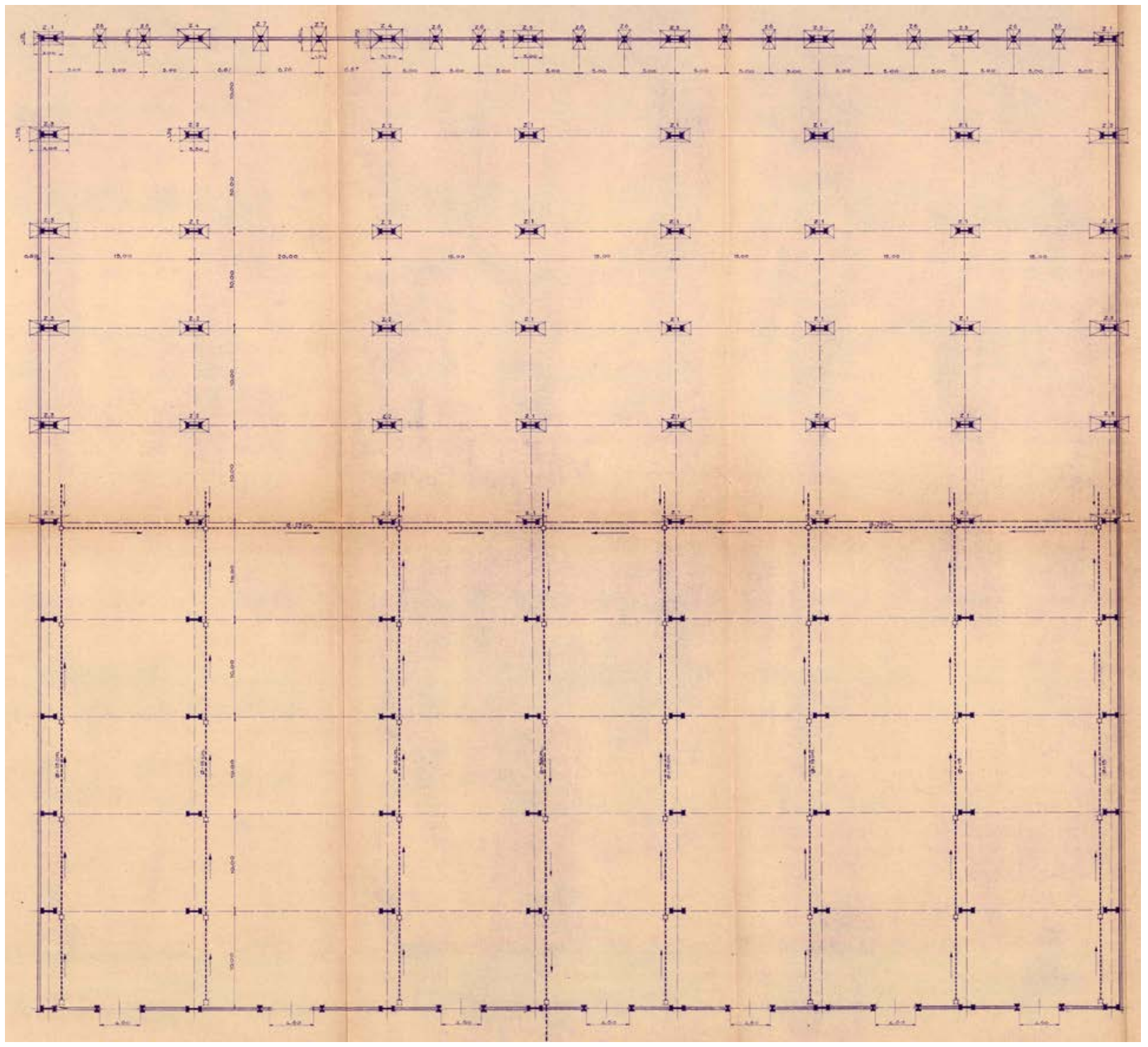

Figura 6. Planta proyecto inicial talleres Gómez Navarro (CEHOPU, ETM-212-001 plano 394.207). 

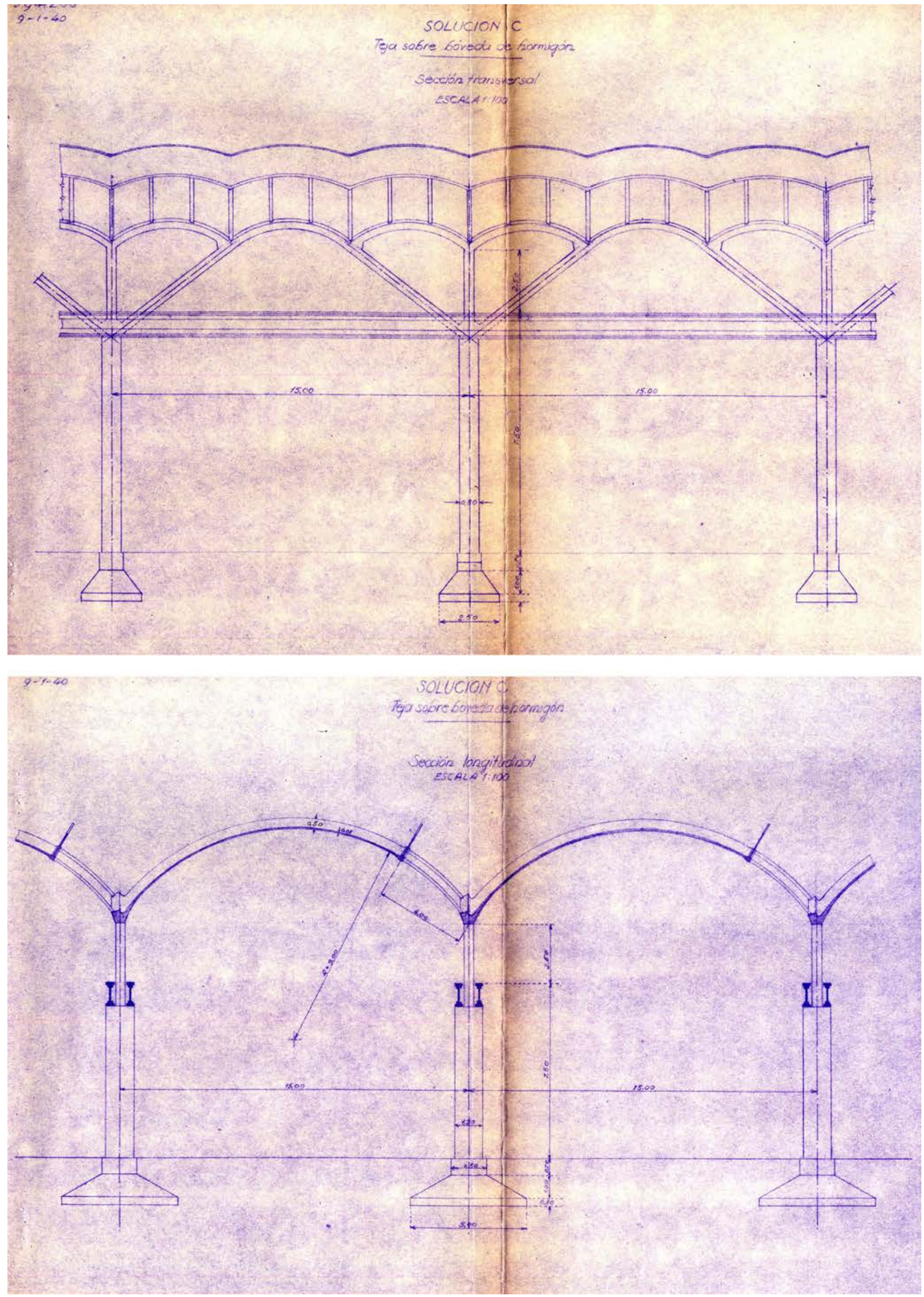

Figura 7. Soluciones de tanteo abovedadas (CEHOPU ETM-212-001 planos 394-205 y 206). 


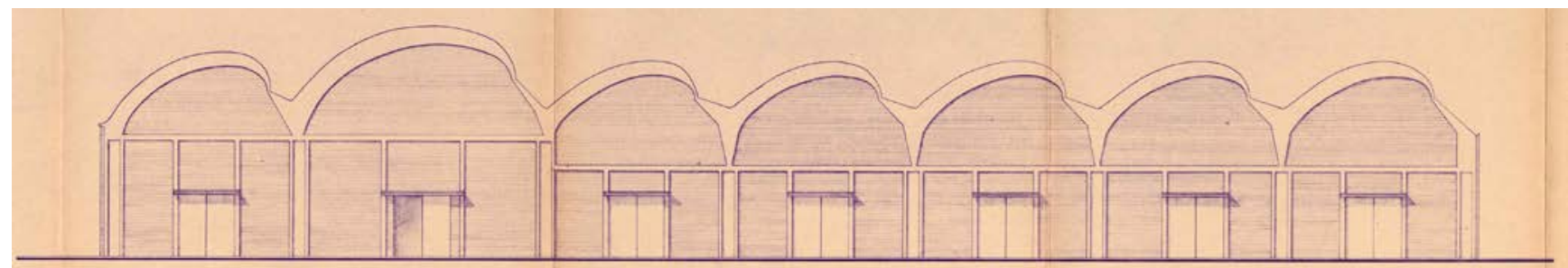

FACHADA

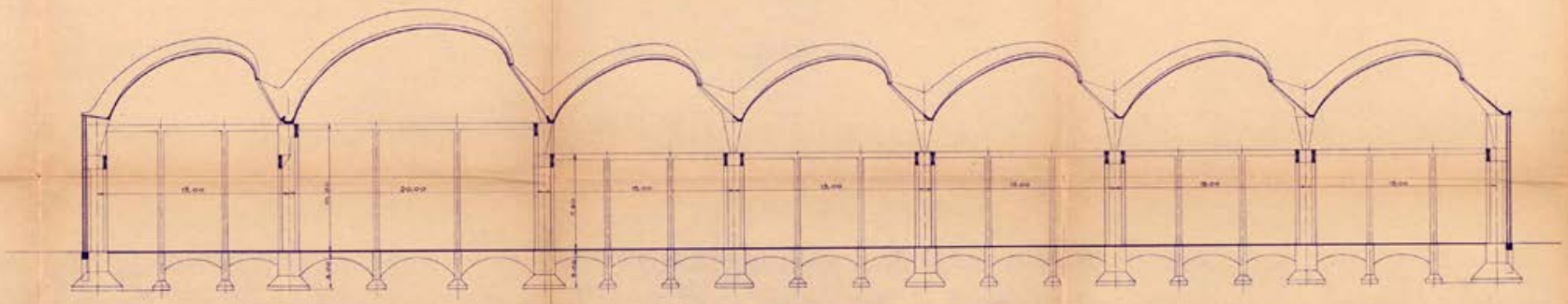

SEC(IÓN TRANSVERSAL

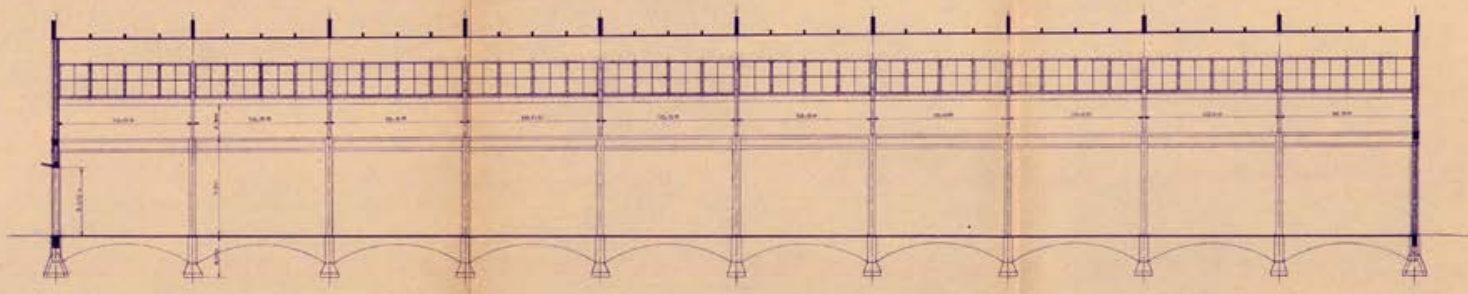

SEC(IÓN LONGITUDINAL

Figura 8. Variante anteproyecto con dientes de sierra arqueados (CEHOPU ETM-212-001 plano 394-208).

la atención que no se haga referencia a la solución estructural que conllevan. El tipo C incluye la solución con cuchillos y también las dos abovedadas.

Prestando algo más de atención a las soluciones con pórticos diente de sierra, se aprecia que consistían en realidad en estructuras de barras rectas o curvas diseñadas de tal forma que cada una apoyaba uno de sus extremos a la altura de la cabeza de los pilares y el otro en el punto de apoyo proporcionado por el apéndice inclinado de la estructura contigua, creándose así una serie de piezas en equilibrio encadenado (Figura 8). El resultado eran grandes elementos en diente de sierra de $15 \mathrm{~m}$ de longitud en los que además, la superficie de cubierta entre pórticos se ubicaba total o parcialmente al nivel del intradós, dejando así una parte o toda la sección de hormigón como un resalte al exterior (Figura 9).

\section{LA NAVE CONSTRUIDA}

La solución realizada fue finalmente la cercha comentada - tal como se indica también en el archivo CEHOPU- lo que significó un cambio radical de planteamiento al sustituir los mó- dulos de diente de sierra de $15 \mathrm{~m}$ de los tanteos previos, por cuatro unidades menores, de $5 \mathrm{~m}$, enlazadas entre sí (Figura 10). La cercha propuesta se repitió sin variaciones en toda la nave dando lugar a luces constantes de $20 \mathrm{~m}$ que la dividen en 6 crujías paralelas, todo ello sobre una planta de dimensiones ligeramente distintas a la de los tanteos previos. La red estructural de soportes es de 20 x 12 m con separaciones de cerchas de $4 \mathrm{~m}$. Un conjunto de vigas continuas de atado de hormigón uniendo las cabezas de los soportes sirve asimismo de apoyo a las cerchas y configura los pórticos longitudinales. Un croquis conservado en CEHOPU (17) parece corresponder a estas vigas, que tendrían entonces un canto de 1,20 $\mathrm{m}$ y por su forma con alojamientos y rebajes sugerirían ser realizadas como prefabricados. A ambos lados de los pilares y a menor altura se dispuso también otro sistema paralelo de vigas de gran canto usadas para los carriles de puentes grúa. Entre ambas vigas los soportes reducen su sección mediante planos inclinados y se puede apreciar que en algunas partes los dos sistemas de vigas están trabados con elementos complementarios para producir un conjunto de arriostramientos de gran rigidez. Estos consisten en gruesos montantes verticales bajo cada cercha y en diagonales entre los recuadros formados por 




Figura 9. Detalle anteproyecto con dientes de sierra rectos (CEHOPU ETM-212-001 plano 394-213).



Figura 10. Cercha proyecto realizado (CEHOPU ETM-212-002 plano 394-220_2).

los anteriores, todo ello en hormigón. Los apoyos de las cerchas se efectuaron de tipo fijo en un extremo y móvil con rodillos en el otro, y su arriostramiento se realizó con viguetas de $40 \times 10 \mathrm{~cm}$, igualmente prefabricadas y situadas en alojamientos en la parte superior e inferior de los planos acristalados. Existe también la singularidad de que en la parte trasera de la nave las vigas de atado-apoyo sobresalen $4 \mathrm{~m}$ en voladizo, soportando en sus extremos la última serie de cerchas, es decir las situadas en coincidencia con el plano de esa fachada trasera. Las vigas del puente grúa tienen también el mismo vuelo. El proyecto conservado en el Archivo de la Villa ofrece así mismo indicaciones sobre la cimentación proyectada:
La cimentación de las naves se ha hecho con pozos rellenos de mampostería con objeto de disminuir el riesgo de ataques del cemento por el yeso que contiene el terreno de cimentación. Los muros van cimentados sobre macizos de mampostería y arcos de descarga del mismo material (18).

Según los datos disponibles se trataría de la primera gran estructura prefabricada de este tipo y dimensiones proyectada en España, ya que todas las referencias apuntan a que la generalización de estas soluciones en nuestro país se produjo algo más tarde. Una interesante fuente de comparación nos la ofrece por ejemplo, la recopilación de las estructuras 


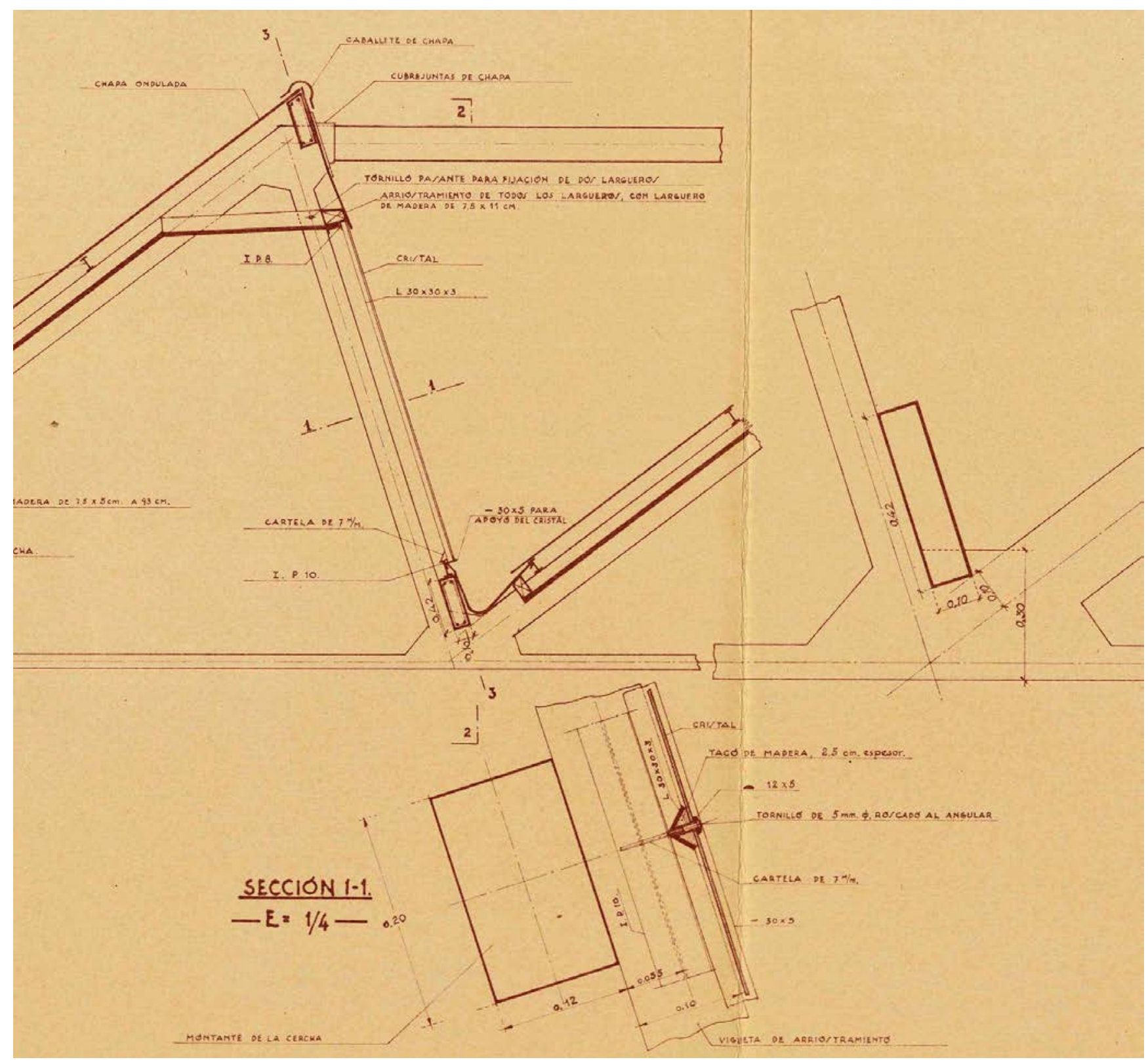

Figura 11. Cercha realizada, detalles (CEHOPU ETM-212-002 plano 394-219_1).

de cubiertas industriales prefabricadas de hormigón realizadas en España publicada entre 1959 y 1960 en tres números de Informes de la Construcción elaborados en colaboración con Huarte y Cía (19) (20) (21). En el primero de ellos se cita como primera cubierta prefabricada realizada por esta empresa, la de la Escuela de Ingenieros de Montes de 1942 en forma de sencilla cercha triangular y luz claramente inferior a $20 \mathrm{~m}$. Después se describen cerchas en diente de sierra dobles y triples en las factorías Fiat de Madrid y Barcelona y en la nave de talleres de Ensidesa. La luz máxima alcanzada en ellas fue de 16,50 m para la Fiat de Madrid de 1951. Las cerchas de Boetticher fueron en cambio realizadas por Agromán S.A y tenían un módulo más que la mayor de las realizadas posteriormente. Su longitud es, como se ha dicho, de $20 \mathrm{~m}$, y sus planos detallados en CEHOPU tienen fecha de siete de marzo de 1941 (22). Por la fecha de inauguración antes indicada se deduce que ya estaban construidas a finales de noviembre de 1942.
Otro aspecto a resaltar es la liviandad con que se realizó esta estructura prefabricada en obra. Las secciones de la cercha oscilan entre 20 x $20 \mathrm{~cm}$ para el cordón superior - con una longitud entre nudos de 4,95 m - y 8 x $20 \mathrm{~cm}$ para el tirante inferior, armado mediante zunchado. A modo de comparación, los cordones superior e inferior de las cerchas de tres vanos publicadas en Informes de la Construcción arriba mencionadas, eran de 40 x 20 y 30 x $20 \mathrm{~cm}$, si bien es cierto que sus separaciones eran de $6 \mathrm{~m}$ frente a los 4 de la nave de Torroja. Con todo, la impresión visual que se tiene es casi la de la ligereza de una cercha metálica. Se moldearon en el suelo y como correas se usaron vigas metálicas de perfil IP 8, disponiendo entre ellas chapa ondulada de zinc con una capa de asilamiento de corcho bajo ella soportada sobre listones de madera (Figura 11). Son ingeniosos y de gran sencillez por otra parte, los detalles de fijación de los cristales observados en los planos de detalle de las cerchas. Para ello se emplean exclusivamente angulares de muy reducida sección, peque- 


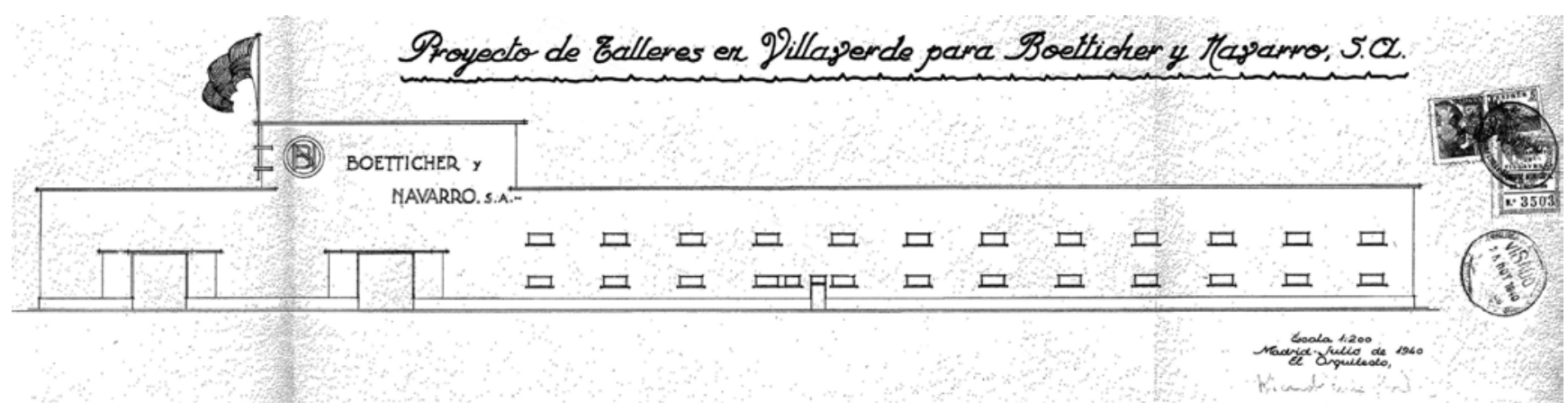

Figura 12. Alzado nave talleres (AVM exp. 33-332-1).

ñas cartelas de chapa y pletinas atornilladas con intermediación de tacos de madera.

Las fachadas exteriores se realizaron con muros de cerramiento de gran simplicidad, lisos en general y con unos resaltes apilastrados en la fachada principal que la dotan de un cierto orden pero que no figuran en los planos (Figura 12). Su destino fue, como se dijo, el de talleres mecánicos de la empresa, mientras que la posterior nave Catedral, con su mayor altura, era la nave de trabajos de fundición. Al exterior de los talleres se proyectaron adosadas las baterías de aseos y otras dependencias formando pequeños cuerpos unidos al rectángulo principal. Interiormente, el espacio era totalmente diáfano y sin particiones, con la excepción de una pequeña entreplanta en fachada destinada a almacén y manifestada en los planos por los dos pisos de ventanas. Del resto de huecos son de señalar los grandes portones este y oeste en la nave de mayor altura y otra gran puerta lateral en la fachada sur para acceso del ramal de ferrocarril que penetraba en el interior de la nave. El hueco de los portones se construyó mediante un pórtico-marco de hormigón de 4,60 de ancho y 4,80 m de alto. Posteriormente se fueron adosando nuevos elementos entre los que puede señalarse una torre sobresaliente en una esquina para pruebas de ascensores, cuando la empresa se reconvirtió en un fabricante de referencia de ellos en España. Esta se realizó según proyecto firmado por el también ingeniero de la empresa Emilio del Corro y con solicitud de licencia de 24 de junio de 1961 (23). Hoy sigue permaneciendo como modesto hito vertical del conjunto siendo utilizada como distintivo de empresa a través del rótulo pintado en ella. (24). No obstante, el añadido que parece más llamativo es la ampliación trasera en una longitud de $44 \mathrm{~m}$ de una de las crujías de 20 m, registrado con petición de licencia de 1947. En ella se dice explícitamente que se repetirá la solución solicitada en la licencia de 19 de noviembre de 1940, es decir la cercha de Torroja. Solo que en este caso los planos, de extrema simplicidad puesto que toman como referente lo ya construido, son firmados por el ingeniero Manuel Cámara Muñoz (25). El cuerpo lateral sur, presumiblemente de oficinas, que cierra hoy la nave en toda esa fachada no estaba tampoco en los planos iniciales.

\section{CONCLUSIONES}

Podría resumirse por tanto, que mientras que la nave Catedral, construida posteriormente, ha sido hasta ahora el centro de atención, en realidad la "verdadera" nave Torroja se encuentra desapercibida y justo a su lado. El desinterés por esta nave contrasta además con la gran atracción mediática suscitada estos últimos años por la mencionada nave Cate- dral o nave Boetticher, lo cual se ha debido, en parte por el debate generado por su reconversión y en parte por lo llamativo de su estructura abovedada de hormigón. Pero tampoco es desde luego carente de belleza el espacio de la nave aquí comentada: una sala hipóstila rectangular de luces y tamaño considerables, dientes de sierra intermedios suspendidos en el aire sin el apoyo de soportes directos, una altura libre constante de 7,35 m, y la excepción de una nave interior más alta con gálibo de $13 \mathrm{~m}$.

Así pues, y frente a la solución estructural abovedada de la nave de fundición, atractiva por su forma pero más bien conservadora dentro de su tipo laminar, la nave de talleres sí fue construida con una solución pionera en España, pese a su exterior de apariencia más convencional. Sobre ella además, puentes grúa de dobles celosías metálicas de $20 \mathrm{~m}$ de luz, con ruedas separadas $3 \mathrm{~m}$ y cargas de hasta 15 toneladas

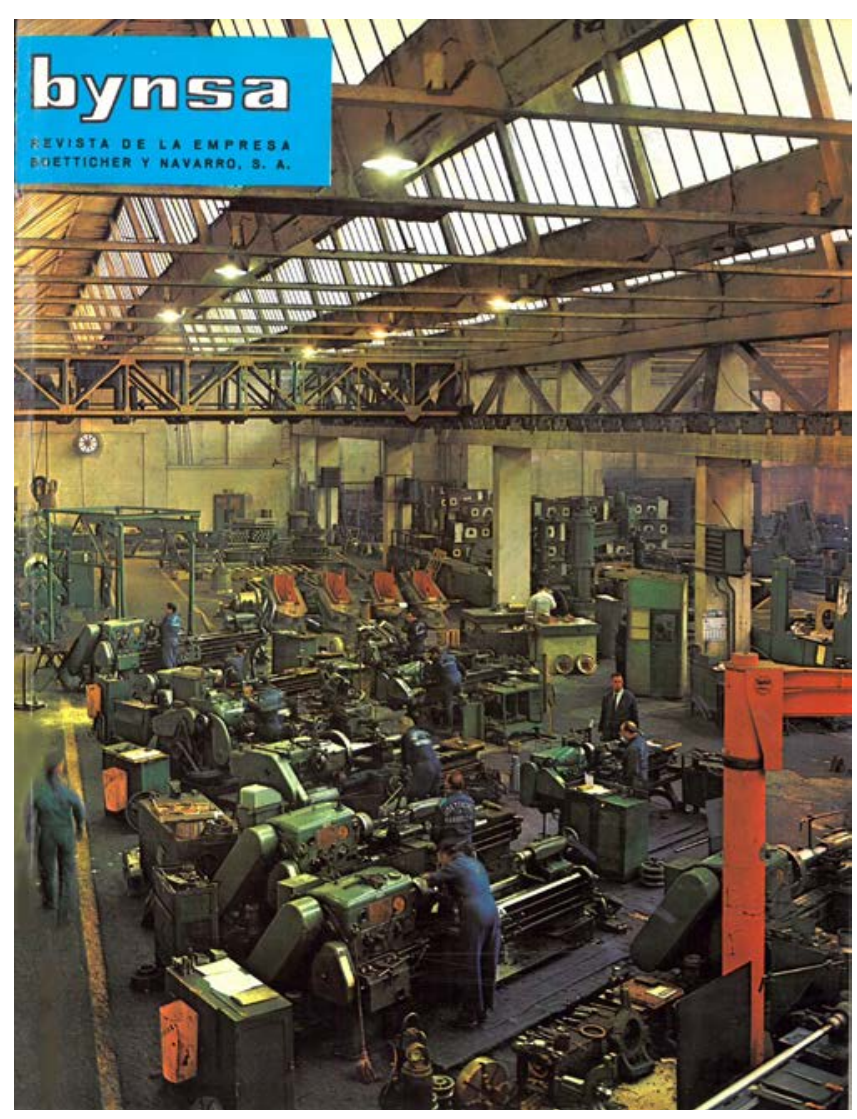

Figura 13. Revista bynsa, época III, (21), III trimestre 1969. Portada con vista interior sección de tornos de la nave. 




Figura 14. Interior de la nave en la crujía de mayor altura. Estado actual (foto del autor).

en cada una de ellas, discurrían libremente por cada una de las seis crujías paralelas. En la publicidad de la empresa fueron incluidas algunas imágenes de sus interiores y también en las páginas de la revista BYNSA, órgano informativo de la empresa entre sus trabajadores (Figura 13). Son, junto con las imágenes aparecidas en la revista del INI, de los pocos testimonios publicados del carácter de su espacio.

Afortunadamente, y a pesar del cambio de uso actual para garaje y talleres de mantenimiento de autobuses, la nave ha sufrido pocas modificaciones, por lo que permanece prácticamente intacta su solución estructural. Al no haberse construido ningún tipo de falso techo dicha solución de cerchas con su sistema de correas es perfectamente visible desde el interior (Figura 14). Se mantienen incluso los puentes grúa metálicos montados sobre sus carriles, aunque no parece que hayan estado operativos desde hace ya bastante tiempo. En las sólidas vigas de apoyo de las cerchas y del puente grúa se aprecian así mismo, los detalles de cajeados y juntas para partes en vuelo sugeridos en algunos de los detalles de planos originales. Así pues, por su buen estado de conservación y por todo lo indicado en el desarrollo de este trabajo, parece justa la reivindicación de esta nave como un elemento patrimonial de importancia al tratarse de una de las estructuras industriales ciertamente valiosas, y por suerte aún existentes, en el entorno madrileño.

\section{REFERENCIAS}

(1) http://www.espinillo.org/foros/foroppp.php?foro=6\&tem=1461\&pagina=13 (último acceso 17/07/2017).

(2) Empresa Boetticher y Navarro s.a. Proyectos de nuevas naves y ampliaciones. AVM 33-306-1.

(3) Proyecto talleres en Villaverde para Boetticher y Navarro, s.a. AVM [Archivo de la Villa de Madrid] 33-332-1.

(4) http://www.espinillo.org/foros/foroppp.php?foro=6\&tem=1349\&pagina=105 (último acceso 18/07/2017).

(5) Martínez de la Madrid, Adelardo. (1948). La creación de zonas industriales en Madrid. Colaboración del Instituto Nacional de Industria en su iniciación. Gran Madrid, (3): 19-25.

(6) INI. (1954). El cincuentenario de Boetticher y Navarro, S.A. INI, 8 (2): 3-13.

(7) Antuña Bernardo, Joaquín. (2002). Las estructuras de Eduardo Torroja y Miret. (Tesis doctoral no publicada). Madrid: Universidad Politécnica de Madrid. Open access en: http://oa.upm.es/1348/1/JOAQUIN_ANTUNA_BERNARDO.pdf

(8) Fernández Ordóñez, José Antonio y José Ramón Navarro Vera (1999). Eduardo Torroja: ingeniero. Madrid, Pronaos.

(9) Jordá, Carmen ed. (2002). Eduardo Torroja, la vigencia de un legado. Congreso. Universidad Politécnica de Valencia.

(10) Levi, Franco ed.(2003). Eduardo Torroja: from the philosophy of structures to the art and science of building: International Seminar, Politecnico di Torino, Franco Angeli. 
(11) Chías Navarro, Pilar ed. (2005). Eduardo Torroja: obras y proyectos. Madrid, Instituto Eduardo Torroja de Ciencias de la Construcción.

(12) Torroja Miret, Eduardo (1999). Las estructuras de Eduardo Torroja / vistas por Eduardo Torroja. Madrid, CEDEX. (Primera edición 1958).

(13) Arredondo y Verdú, Francisco (1977). La obra de Eduardo Torroja. Madrid, Instituto de España.

(14) Colegio Oficial de Ingenieros de Caminos, Canales y Puertos de Madrid (1977). Madrid, editorial Turner.

(15) Planta de estudios previos. CEDEX-CEHOPU ETM-21-001/Caja 057/03, n 394.207.

(16) Estudios previos CEDEX-CEHOPU ETM-21-001/Caja 057/o3, ns. 394.205, 394.206, 394.208, 394.209, 394.210, 394.211, 394.212, 394.213, 394.214, 394.215.

(17) Croquis acotado jácena puente grúa CEDEX-CEHOPU ETM-21-002/Caja 057/04 n.394.524.

(18) Proyecto de talleres en Villaverde para Boetticher y Navarro. Memoria. AVM 33-332-1.

(19) Informes. (1959). Prefabricación I. Informes de la Construcción (113).

(20) Informes. (1959). Prefabricación II. Informes de la Construccion (114).

(21) Informes. (1960). Prefabricación III. Informes de la Construcción, (118).

(22) Cercha proyecto definitivo CEDEX-CEHOPU ETM-21-002/Caja 057/o4 ns. 394.219 y 394.220.

(23) bynsa. (1954). Lista de personal técnico (en trasera). Revista de Boetticher y Navarro S.A. (1).

(24) Torre de pruebas de ascensores en Boetticher y Navarro. AVM 990-877-052242.

(25) Empresa Boetticher y Navarro s.a. Ampliación nave talleres. AVM 33-306-1. 